\title{
Thyroid Cancer in the Pediatric Population
}

\author{
Silva Frieda, Nieves-Rivera Francisco and Laguna Reinaldo \\ University of Puerto Rico, School of Medicine, San Juan, \\ Puerto Rico
}

\section{Introduction}

Thyroid Cancer is the third most common solid tumor in children, accounting for $35 \%$ of the carcinomas in pediatric populations. The reported incidence in the United States is relatively low compared to other tumors (approximately 0.2-0.5 per 100,000/year) with a reported age adjusted incidence of 8.5 per 100,000. As in adults, the tumors are more frequent in females. The female to male incidence ratios vary with age. In children under the age of 10 the ratio is $1.2: 1$, in the 10-21 year old group it is 3.6:1(Shapiro, 2005). Overall, $10 \%$ of all cases occur in patients younger than 21 years and the reported average age at presentation is 11-19 years (Shapiro, 2005, Parisi, 2007).

The initial clinical presentation of pediatric thyroid cancer is usually an asymptomatic mass in the neck. Thirty three to fifty percent of those identified neck masses are subsequently diagnosed as malignant. Certain characteristic features, such as firmness of the mass and the degree of fixation to surrounding tissues, as well as lymphadenopathy and/or vocal cord paralysis are associated with an increased probability of malignancy. Pain, tenderness, difficulty swallowing and respiratory problems are not typically reported in the majority of pediatric patients (Jarzab, 2005). Important risk factors for the development of thyroid cancer in children include exposure to external radiation, congenital hypothyroidism and a history of prior malignancies (Parisi, 2007, Niedziela, 2006).

The location of well-differentiated tumors (WDTC) in the gland is variable; however the majority $(68 \%)$ of the reported malignancies are localized in the right lobe. Children with thyroid tumors are generally clinically euthyroid (Jarzab, 2005).

The four main histologic types of pediatric thyroid cancers are papillary, papillary-follicular variant, follicular (FTC), and medullary carcinoma. The majority of the tumors (90-95\%) are classified as well differentiated type, i.e., papillary and follicular type (Niedziela, 2006). Medullary tumors are diagnosed in 5-8\% of the patients and produce calcitonin. Medullary carcinomas arise from the parafollicular cells or $C$ cells while the papillary and follicular tumors originate from the follicular epithelium. Medullary tumors will be discussed later in the chapter.

Papillary thyroid tumors (PTC) are larger in pediatric patients as compared to tumors in adults at presentation. Capsular invasion is seen in $67 \%$ of the pediatric patients (Parisi, 2007, Niedziela, 2006). Unlike adults, pediatric patients often present with extensive regional nodal disease and distant metastasis at diagnosis. Cervical nodal disease ranges from 60- 
$90 \%$; distant metastasis occurs in 13-23\%, most commonly to the lung (Parisi, 2007, Jarzab, 2005). Tracheoesophageal nodes are the ones most frequently involved, followed by the mid jugular and the lower deep jugular group. Bulky nodal metastasis may be associated with small tumors, $1 \mathrm{~cm}$ or less. Lung metastasis occurs more frequently in patients with extra capsular invasion, bilateral tumors and patients below age 7 at diagnosis. Pulmonary metastases in children are typically miliary and rarely nodular as opposed to adults. Metastases to the lungs are functional in 95\% of the cases (Luster, 2007, Mitsutake, 2005). This is related to greater expression of the sodium iodide symporter (NIS) in pediatric thyroid cancers. The increased expression of NIS in pediatric tumors implies a greater degree of differentiation compared to adult papillary cancers. Long-term survival is also poorer; however the reported difference in survival is narrow (Shapiro, 2005).

\section{Diagnosis}

Thyroid tumors are suspected by a history of an anterior neck palpable mass with or without hoarsenes. Further imaging evaluation studies should include thyroid ultrasound which is the method of choice for the assessment of nodular disease. It is a rapid, safe, noninvasive, and readily available technique. In addition, sonographic evaluation helps to distinguish cystic from solid lesions and allows guidance for fine needle aspiration biopsy (FNA). The sonographic appearance of thyroid cancer varies with the histologic type. PTC lesions are usually solid and hypoechoic with (i.e., $70 \%$ of the affected) increased vascularity and micro calcifications. The presence of microcalcifications is highly specific for PTC. On the other hand, FTC is more frequently iso- or hyperechoic with a thick and irregular halo. The latter appearance associated with the presence of suspicious regional lymph nodes increases the risk of malignancy in pediatric thyroid nodules. However, the presence of cystic lesions should not be ignored since $50 \%$ of all malignant thyroid lesions have a cystic component and $8 \%$ of sonographic cystic lesions represent neoplasia (Niedziela, 2006,Jarzab, 2005,Luster, 2007).

Presently ultrasound-guided fine needle aspiration (FNA) is the most reliable and cost effective method to establish a cytologic diagnosis in up to $73 \%$ of lesions prior to surgery. Specifically, a true positive diagnosis can be obtained in up to $90 \%$ of the lesions with PTC. However, in children FNA accuracy may be less than in adults as reported by Parisi \& Niedziela. Therefore, a negative FNA must be interpreted with caution and it should not be used as the sole predictor of malignancy.

Nuclear thyroid scintigraphy with $99 \mathrm{mTc}$-pertechentate or 123-Iodine may be used in the initial evaluation. It helps in the identification of the "cold nodule", a lesion described as a well-defined area with less radiotracer uptake as compared to normal tissue. It must be remember that only $10-15 \%$ of cold lesions in a thyroid scintiscan are malignant, meaning that sonographic guided FNA is needed for a definite diagnosis. In addition, lesions less than $1 \mathrm{~cm}$ may be missed, an important limitation of nuclear thyroid imaging to bear in mind.

Immunocytochemical studies are used to further improve the diagnosis. Several tumor markers have been studied in an attempt to enhance diagnosis. Among these are telomerase, galactin-3 and cytokeratin-19. Unfortunately, none have been able to distinguish benign from malignant lesions in children, particularly in cases of follicular tumors or follicular variant of papillary thyroid cancer (Xu, 2003, Mitsutake, 2005). 
Recently, interest has focused on genetic markers as a diagnostic tool since they might have a role in terms of tumor behavior. Kimura et al (2003) studied 177 cases of PTC and found RET, NTRK, BRAF and/or RAS mutations in $70 \%$ of the tumors. Namba et. al. (2003) reported that papillary lesions with BRAF gene mutation have more aggressive extrathyroidal invasion with less favorable prognosis. This is more frequently seen in the columnar type and the classic PTC variant (Xu, 2003, Mitsutake, 2005). It leads to the origin of oncogene BRAF which activates MAPK kinase. Activation of this cascade has the effect of inappropriate cell proliferation and differentiation into neoplasia.

Other investigators have reported over expression of MET in papillary cancers. Together with the tyrosine kinase receptor ligand it is associated to a higher recurrence risk in children. Gupta (2001) et al found that patients with PTC with the greatest number of proliferating lymphocytes in thyroid infiltrates have the longest disease free survival (Jarzab, 2005, Griffith 2006). On the other hand, patients with intense expression of B7-2 antigen had a greater propensity for recurrence (Jarzab, 2005). Kroll et. al. identified a mutation in follicular thyroid cancers - twenty to fifty percent of them harbor an interchromosomal translocation that fuses PAX8 to PPARY. This acts as an oncoprotein. FTC that do not have PAX8 / PPARY recombination are often associated with RAS mutations (Xu, 2003,Mitzutake, 2005,Griffith, 2006).

\section{Tumor staging}

The American Thyroid Association (ATA) published in 2009 the guidelines for the management of thyroid nodes and differentiated thyroid cancer. Using the TNM classification pediatric thyroid cancer is classified as a TNM stage I or II, according to the presence or absence of distant metastasis regardless of the existence of lymph node metastases.

It is recognized that pediatric thyroid cancer at diagnosis often involves spread to lymph nodes as well as distant metastasis. Based on these characteristics at diagnosis, some authors have recommended the use of a sub stage classification that takes into consideration major risk factors such as gender, multifocality, lymph node invasion, distant metastasis and young age. Based on the frequency of extensive disease, lymph node and the presence of distant metastasis and the high recurrence rate, most children should be included in the high risk group, even when most staging systems classify them in Stage 1 or Stage 2 (Wada, 2009).

\section{Surgical treatment}

Treatment of WDTC is intended to eradicate disease and improve recurrence free survival. The primary treatment modality remains surgical excision followed by radioiodine ablation and thyroid hormone suppression therapy; a paradigm followed in many institutions.

The extent of surgical removal is still a matter of debate in many places. The ATA and the American Association of Clinical Endocrinologists have recommended a total or near total thyroidectomy if feasible in most of the cases (Cooper, 2009). Total thyroidectomy remains the preferred operation in tumors greater than T1a $(>1 \mathrm{~cm})$. It is generally accompanied by dissection of the central lymphatic compartment since local lymph node disease increases 
the risk of local and regional recurrence (Parisi, 2007, Niedziela, 2006, Handkiewicz, 2007). Patients who undergo total thyroidectomy have a higher rate of recurrence-free survival. Patients with intrathyroidal lesions less than $1 \mathrm{~cm}$ can be considered candidates for less than total thyroidectomy if the lesion is unifocal, there is no previous history of radiation exposure, and there is no evidence for either lymph node or distant metastasis at diagnosis.

Complications of surgery are rare in the hands of experienced surgeons. The most common complication is parathyroid dysfunction, resulting in hypocalcemia and low serum PTH concentrations. The hypoparathyrodism is usually transient and resolves in several weeks with some patients requiring temporary treatment. A more serious complication is the irreversible damage to the recurrent laryngeal nerve causing permanent vocal cord paralysis and hoarseness; however, in our practice we have never seen such a case. Nowadays the use of intraoperative probe reduces surgical time and complications.

\subsection{Post-surgical evaluation and radioiodine treatment}

A sonographic evaluation is advisable to assess the size of the thyroid remnant and determine the adequacy of excision after surgery. Images should depict a volume of less than $2 \mathrm{ml}$ of thyroid tissue at approximately 1 month after its removal.

Thyroid ablation with radioiodine (RAI) is routinely recommended to destroy any residual tissue after surgery. The goal of the postsurgical treatment is to ablate the thyroid remnant and evaluate the extent of functional thyroid tissue and/or metastatic disease. The radioiodine dose should be individualized and is often empirically adjusted to tumor size and disease extension. Therefore, RAI doses ranging from 30-200 mCi (1.1-7.4 GBq) have been employed. The use of low dose vs. high dose treatments is still a debated issue, since some authors claim there is little difference in efficacy between the $30 \mathrm{mCi}$ and the $100 \mathrm{mCi}$. The higher activity is more effective in detecting and treating metastatic disease. In our practice we use doses in the pediatric population similar to those in adults. For patients with disease extension to neck nodes we recommend 100mCi (3.7 GBq) and for patients with distant metastasis we have used 150-200 mCi (5.6-7.4 GBq). We pursue Whole Body Scan (WBS) post therapy usually 5-7 days after the therapeutic radioiodine dose. In most patients, the WBS demonstrates post surgical residual functioning tissue in the thyroid bed, additional loco-regional nodes or distant metastases. Post therapy scintigraphy may detect new lesions in up to $46 \%$ of the cases (Silva, 2010).

Thyroid ablation therapy reduces the risk of recurrence in the thyroid bed and neck lymph nodes, independent of the extent of the surgical procedure. Both treatment modalities (surgery and RAI ablation) are independent predictors of recurrence free survival. Local relapse is reduced to $6.3 \%$ when 131-I is administered after surgery. Recurrence rate in the thyroid bed and local lymph nodes is also reduced when total thyroidectomy and ablation therapy are given. This is especially important in the pediatric population given their longer life expectancy. It has been found that sex, age and histology do not correlate with thyroid bed recurrence free survival. Nodal recurrences are more frequent in patients with the histologic subtype of papillary carcinoma, classical variant as compared to the follicular variant. Specifically, the 10 year nodal recurrence free survival has been reported at $83 \%$ for papillary carcinoma, classic type vs. 95\% for the follicular variant type (Parisi, 2006, Luster, 2007, Handkiewicz, 2007). 
In patients with lung metastasis, there is often incomplete post therapy remission. Several authors have reported a high incidence of persistent disease after therapeutic iodine in patients with lung metastasis. Repeated courses of ablation therapy is often needed. Since ablation therapy could have adverse effects on the lungs (i.e., pneumonitis with fibrosis), it is important to monitor pulmonary function in this population.

The tumor response to RAI therapy is related to the size of the residual tissue or tumor burden. Tumoral masses with small volume (i.e., less than $1 \mathrm{~mm}$ ) in children results in poorer therapeutic response. This is related to the effect of the iodine beta radiation range, as established in the Monte Carlo simulation test published by Reynolds, Robbins and Maxon. Approximately $90 \%$ of the ionizing radiation emitted by the 131-iodine decay will be absorbed outside the tumor range (Silva, 2010).

Juvenile thyroid cancer is known to have a high recurrence rate. The rate may be as high as $40 \%$ in patients less than 20 years of age and higher within the first 7 years of diagnosis. Twenty year recurrence free survival is about $10 \%$ in patients diagnosed at age 10 or less. In fact, age is a major determinant of risk of recurrence, particularly in those younger than 10 years (Niedziela, 2006, Jarzab, 2005). Overall younger patients have a poorer prognosis than those diagnosed later in life. In spite of these data, it should be emphasized that prognosis is still excellent with an overall survival rate of $95 \%$ for over a 12.9 years of follow up in large series (Shapiro, 2005, Parisi, 2007).

Five and ten year survival rates in pediatric patients with thyroid cancer have been reported as high as $98-99 \%$. However, the mortality rate may become significant in cases with distant metastasis or recurrent disease. In the latter, the cumulative mortality rate varies from 30$58 \%$ in a period of $12-20$ years.

Over the last several years, FDG-PET studies have been included in the armamentarium of diagnostic tests in the follow up of patients with thyroid cancer. PET imaging is recommended in selected cases only, specifically those with negative iodine WBS and positive or rising thyroglobulin levels. FDG uptake can identify recurrent disease in up to $70-80 \%$ of those cases; uptake is associated with a poor outcome and decreased survival (Parisi, 2007).

\section{Risks of radioiodine therapy in the pediatric population}

Radioiodine therapy can have complications, both in the short and in the long term. Short term complications include nausea, sialadenitis, pain and swelling in the neck. The frequency of these varies from $10-50 \%$. Long term effects are related to the radiationinduced mutations (i.e., radiation carcinogenesis). These are of utmost importance given the long life expectancy the pediatric group has at the time of diagnosis.

In terms of the gonadal axis, RAI therapy is associated with an earlier menopause compared with the general female population. In males azoospermia and oligospermia have been described. Increased risks of secondary malignancies, mainly solid tumors and leukemias, have also been reported. Pulmonary fibrosis has been reported in patients with extensive pulmonary metastasis; in fact up to $10 \%$ of pediatric patients can develop pulmonary fibrosis. All of the above complications are dose dependent (Parisi, 2007). 


\section{Follow up}

Hormonal suppressive therapy follows the ablation therapy and is aimed at reducing the risk of tumor re growth and recurrences. The recommended TSH suppression level may be in the range of $0.1-0.5 \mathrm{mU} / \mathrm{L}$ and should be dependent on the presence or absence of persistent disease.

Serum thyroglobulin (Tg), a tumor specific marker, is a valuable assay during follow up. $\mathrm{Tg}$ is only produced by the follicular cells. After thyroidectomy, Tg should be undetectable. The assay of thyroglobulin is recommended before ablation therapy (under TSH stimulation) and 6-12 months after therapy (on suppression). The predictive value for disease progression is well established in the literature.

Stimulated levels of serum thyroglobulin more than 2 or $3 \mathrm{ng} / \mathrm{ml}$ after recombinant TSH are considered diagnostic of tumor recurrences. A rising value of $\mathrm{Tg}$ alerts the clinician to the presence of active disease and is more sensitive if the sample is measured after TSH stimulation. It is recommended to have the samples analyzed in the same laboratory using the same assay method. Thyroglobulin antibodies ( $\mathrm{TgAb})$ must be determined in the same serum sample since the presence of $\mathrm{TgAb}$ invalidates the thyroglobulin results. (Goldsmith, 2011).

Some authors have reported that $\mathrm{Tg}$ use is not completely reliable for follow up purposes. Specifically, they do not advocate the sole use of $\mathrm{Tg}$ as a method for follow up in affected patients when screening for persistent or recurrent disease. False negative Tg values have been reported in $4-35 \%$ of the patients with evidence of local or metastatic disease. Undetectable serum $\mathrm{Tg}$ has been reported in patients treated with RAI, with small volumes of disease. Other reasons associated with the low or undetectable Tg levels are the presence of $\operatorname{TgAb}$, immunologically inactive $\mathrm{Tg}$ or technical limitations of the assay method. Thus, relying on Tg values as a single criterion in the long term follow up of thyroid cancer should be exercised with caution. In our center, pediatric patients are evaluated annually for at least three consecutive years and thereafter every two or three years depending on the clinical follow up evaluation. All patients are evaluated withTg and Tg Ab levels as well as a neck ultrasound. Neck ultrasound is an excellent tool for the early detection of neck nodal metastasis and should be used in conjunction with the Tg measurement in the follow up of patients whenever possible (Parisi, 2007, Niedziela, 2006, Jarzab, 2005). In our clinic, patients with initial distant metastasis, 131-I Whole body scan is done one year after the therapeutic dose to determine if an adittional therapeutic doses is necessary. Clinical follow up is recommended until adulthood.

\section{Medullary Thyroid Carcinoma}

Medullary thyroid carcinoma (MTC) originates in calcitonin-producing cells (C-cells) of the thyroid gland and accounts for $3 \%$ to $9 \%$ of thyroid cancers. MTC is diagnosed histologically when nests of C-cells appear to extend beyond the basement membrane and infiltrate and destroy thyroid follicles. At least half occur in kindreds and are apparently inherited as an autosomal dominant trait. It may be part of multiple endocrine neoplasia type 2 (MEN 2) including the phenotypes MEN 2A, familial medullary thyroid carcinoma (FMTC), and MEN 2B. 
MTC in persons with MEN 2 typically present at a younger age than sporadic MTC and is more often associated with C-cell hyperplasia as well as multifocality or bilaterality. Symptoms of MTC include neck pain, a palpable neck mass, and/or diarrhea resulting from hypercalcitoninemia [Callender et al 2008]. Metastatic spread to cervical and regional lymph nodes (i.e., parathyroid, paratracheal, jugular chain, and upper mediastinum) or to distant sites including the liver, lungs, or bone is common and is frequently present in individuals with a palpable thyroid mass or diarrhea [Cohen \& Moley 2003].

The responsible mutated gene causing inherited MTC is the ret tyrosine kinase which has been mapped to $10 \mathrm{q} 11.2$.

The FMTC subtype constitutes approximately $10 \%-20 \%$ of cases of MEN 2. By operational definition MTC is the only clinical manifestation of FMTC. The age of onset of MTC is later in FMTC and the penetrance of MTC is lower than that observed in MEN 2A and MEN 2B [Eng et al 1996, Machens et al 2001, Machens \& Dralle 2006, Zbuk \& Eng 2007, American Thyroid Association Guidelines Task Force 2009].

The MEN 2B subtype accounts for approximately 5\% of cases of MEN 2. MEN 2B is characterized by the early development of an aggressive form of MTC in all affected individuals [Skinner et al 1996]. Individuals with MEN 2B who do not undergo thyroidectomy at an early age (age $<1$ year) are likely to develop metastatic MTC at an early age. Prior to intervention with early prophylactic thyroidectomy, the average age of death in individuals with MEN 2B was 21 years.

The American Thyroid Association Guidelines Task Force has classified mutations based on their risk for aggressive MTC [American Thyroid Association Guidelines Task Force 2009]. The classification may be used in (1) predicting phenotype and in (2) recommendations regarding the ages at which to (a) perform prophylactic thyroidectomy and (b) begin biochemical screening for pheochromocytoma and hyperparathyroidism. Annual biochemical screening for MEN 2A beginning at age eight years has been recommended for individuals with mutations of codons 630 and 634 and at age 20 years for mutations in all other codons [American Thyroid Association Guidelines Task Force 2009]. Screening for FMTC is indicated just as for patients in kindreds with MEN 2. MEN 2B patients identified by genetic screening should undergo thyroidectomy at 6 months of age. However screening should not be precluded and still be pursued early in life in children at risk of carrying MEN $2 \mathrm{~B}$ genes since disease manifestation could be aggressive and devastating. The best time for surgery should be individualized (author personal experience). A basal or stimulated calcitonin level of $\geq 100 \mathrm{pg} / \mathrm{ml}$ is an indication for surgery [Costante et al 2007, American Thyroid Association Guidelines Task Force 2009].

The standard treatment for MTC is surgical removal of the thyroid and lymph node dissection [American Thyroid Association Guidelines Task Force 2009]. Chemotherapy and radiation are less effective in the treatment of MTC than surgical removal [Moley et al 1998, Cohen \& Moley 2003]. All individuals who have undergone thyroidectomy need thyroid hormone replacement therapy. Autotransplantation of parathyroid tissue is not typically performed at the time of thyroidectomy unless there is evidence of hyperparathyroidism [American Thyroid Association Guidelines Task Force 2009]. Prophylactic thyroidectomy is the primary preventive measure for individuals with an identified germline RET mutation [American Thyroid Association Guidelines Task Force 2009]. Prophylactic thyroidectomy is 
safe for all age groups; however, the timing of the surgery is controversial. According to the consensus statement from the American Thyroid Association Guidelines Task Force, the age at which prophylactic thyroidectomy is performed can be guided by the codon position of the RET mutation. These guidelines continue to be modified as more data becomes available.

For all individuals with a RET mutation who have not had a thyroidectomy, annual biochemical screening with calcitonin is recommended with immediate thyroidectomy if results are abnormal [Szinnai et al 2003]. Annual serum calcitonin screening [American Thyroid Association Guidelines Task Force 2009] should begin for children with: MEN 2B at age six months and MEN 2A or FMTC at age three to five years. Caution should be used in interpreting calcitonin results for children younger than age three years, especially those younger than age six months. Prophylactic thyroidectomy should not be offered routinely to at-risk individuals in whom the disorder has not been confirmed. RET gene molecular genetic testing should be offered to probands with any of the MEN 2 subtypes and to all atrisk members of kindreds in which a germline RET mutation has been identified in an affected family member.

Alternate therapies are still under investigation with potential benefits in the near future. Among these: Adenoviral vectors expressing a dominant-negative truncated form of RET, DeltaTK; potential of tyrosine kinase inhibitors, such as vandetanib.

\section{Conclusion}

Well differentiated is the most common pediatric thyroid cancer and usually presents as a solitary mass. The disease is frequently extensive at diagnosis. Surgical management is important for the initial control of the disease, follow by radioiodine therapy. High therapeutic radioiodine doses are required in the majority of the cases. Long term follow up with hormonal suppressive therapy is recommended. Neck sonogram and serum thyroglobulin level is recommended for the first 3 consecutive years. In spite of the extensive disease, overall long term survival in these patients is excellent.

\section{References}

American Thyroid Association Guidelines Task Force; Medullary thyroid cancer: management guidelines of the American Thyroid Association. Thyroid. 2009;19:565612.

Cooper D, Doherty G, Haugen B, et al. Revised American Thyroid Association management guidelines for patients with thyroid nodules and differentiated thyroid cancer. Thyroid: 19 No 11, 2009

Callender GG, Rich TA, Perrier ND. Multiple endocrine neoplasia syndromes. Surg Clin North Am. 2008;88:863-895.Cohen MS, Moley JF. Surgical treatment of medullary thyroid carcinoma. J Intern Med. 2003;253:616-26

DeLellis RA, Lloyd RV, Heitz PU, Eng C (2004) Pathology and Genetics: Tumours of the Endocrine Organs. World Health Organization Classification of Tumours series, vol 8. IARC Press, Lyon, France. 
Eng C, Clayton D, Schuffenecker I, Lenoir G, et. al. The relationship between specific RET proto-oncogene mutations and disease phenotype in multiple endocrine neoplasia type 2. International RET mutation consortium analysis. JAMA. 1996;276:1575-9.

Goldsmith S. To ablate or not to ablate: Issues and evidence involved in 131-I ablation of residual tissue in patients with differentiated thyroid carcinoma. Semin Nucl Med 41:96-104, 2011

Griffith O, Melk A, J, Wiseman S. Meta analysis and Meta review of thyroid cancer gene expression profiling studies identifies important diagnostic biomarkers. Journal of Clinical Oncology Vol. 24 No 31: 5043-5051, 2006

Handkiewicz D, Wloch J, Roskosz J et al. Total thyroidectomy and adjuvant radioiodine treatment independently decrease locoregional recurrence risk in childhood and adolescent differentiated thyroid cancer. J Nuclear Medicine 48: 879-888, 2007

Jarzab B, Handkiewicz D, Wloch J. Juvenile differentiated thyroid carcinoma and the role of radioiodine in its treatment: a quantitative review. Endocrine related Cancer 12: 773803,2005

Luster M, Lassmann M, Freudenberg L, Reiners C. Thyroid cancer in childhood: Management strategy, including dosimetry and long term results. Hormones 6(4): 269-278, 2007

Machens A, Gimm O, Hinze R, Hoppner W, Boehm BO, Dralle H. Genotype-phenotype correlations in hereditary medullary thyroid carcinoma: oncological features and biochemical properties. J Clin Endocrinol Metab. 2001;86:1104-9.

Machens A, Schneyer U, Holzhausen HJ, Raue F, Dralle H. Emergence of medullary thyroid carcinoma in a family with the Cys630Arg RET germline mutation. Surgery. 2004;136:1083-7.

Mitsutake N, Knauf J, Nisutake S. et al. Conditional BRAF expression induces DNA synthesis, apoptosis, dedifferentiation and chromosomal instability in thyroid PCCL3 cells. Cancer Research 65(6): 24652473, 2005

Niedziela M. Pathogenesis, diagnosis and management of thyroid nodules in children. Endocrine-Related Cancer 13: 427-453, 2006

Parisi M, Mankoff D. Differentiated Pediatric Thyroid Cancer: correlates with adult disease, controversies in treatment. Seminars in Nuclear Medicine 37:340-356, 2007

Shapiro N, Bhattacharyya N. Population Based Outcomes for Pediatric Thyroid Carcinoma. Laryngospe 115:337-340, 2005

Silva F, Laguna R, Nieves-Rivera F. Pediatric thyroid cancer with extensive disease in a Hispanic population: Outcome and long term survival. Journal of Pediatric Endocrinology and Metabolism 23: 59-64, 2010

Wada N, Sugino K, Mimura T et al. Pediatric differentiated thyroid carcinoma in stage 1: risk factor analysis for disease free survival. BMC Cancer 2009, 9:306

$\mathrm{Xu}$ X, Quiros R, Gatusso P, et al. High prevalence of BRAF gene mutation in papillary thyroid carcinoma and thyroid tumor cell lines. Cancer Research 63: 4561-4567, 2003 
Zbuk KM, Eng C. Cancer phenomics: RET and PTEN as illustrative models. Nat Rev Cancer. 2007; 7:35-45. 


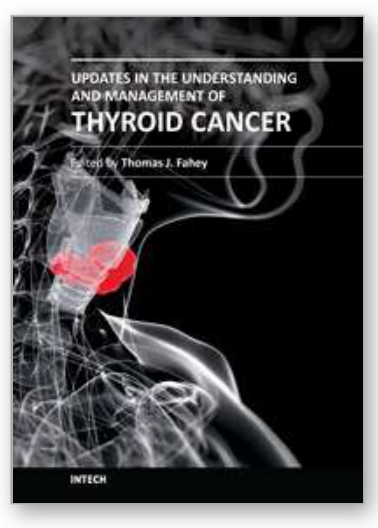

\author{
Updates in the Understanding and Management of Thyroid Cancer \\ Edited by Dr. Thomas J. Fahey
}

ISBN 978-953-51-0299-1

Hard cover, 306 pages

Publisher InTech

Published online 21, March, 2012

Published in print edition March, 2012

\title{
How to reference
}

In order to correctly reference this scholarly work, feel free to copy and paste the following:

Silva Frieda, Nieves-Rivera Francisco and Laguna Reinaldo (2012). Thyroid Cancer in the Pediatric Population, Updates in the Understanding and Management of Thyroid Cancer, Dr. Thomas J. Fahey (Ed.), ISBN: 978-953-51-0299-1, InTech, Available from: http://www.intechopen.com/books/updates-in-theunderstanding-and-management-of-thyroid-cancer/thyroid-cancer-in-the-pediatric-population

\section{INTECH}

open science | open minds

\section{InTech Europe}

University Campus STeP Ri Slavka Krautzeka 83/A 51000 Rijeka, Croatia Phone: +385 (51) 770447 Fax: +385 (51) 686166 www.intechopen.com

\section{InTech China}

Unit 405, Office Block, Hotel Equatorial Shanghai No.65, Yan An Road (West), Shanghai, 200040, China 中国上海市延安西路65号上海国际贵都大饭店办公楼405单元 Phone: $+86-21-62489820$

Fax: +86-21-62489821 
(C) 2012 The Author(s). Licensee IntechOpen. This is an open access article distributed under the terms of the Creative Commons Attribution 3.0 License, which permits unrestricted use, distribution, and reproduction in any medium, provided the original work is properly cited. 